\title{
Intelligent Decision Making System for Car Parking
}

\author{
G. Revathi \\ M.E Pervasive Computing \\ Technologies, \\ Anna University of Technology, \\ Tiruchirappalli-620024, \\ Tamil Nadu, India
}

\author{
V.R. Sarma Dhulipala \\ Assistant Professor (Physics), \\ Centre for Convergence of \\ Technologies, \\ Anna University of Technology, \\ Tiruchirappalli-620024, \\ Tamil Nadu, India
}

\author{
G.R.Kanagachidambaresan \\ M.E Pervasive Computing \\ Technologies, \\ Anna University of Technology, \\ Tiruchirappalli-620024, \\ Tamil Nadu, India
}

\begin{abstract}
In this paper, we explore the real time concept of a smart parking system purported for Geographical Area Considered (GAC) environment. The monetary value of the commercially available smart parking system is eminent. The reliability and efficiency were increased considerably in our proposed work. We use the image-processing concept for identification of the number plate. We make use ZigBee protocol for conveys the information between the entrances and parking zone. The parking system mainly focused on allocating slots and provides slots for car parking. This can be extended by analyzing the human behavior.
\end{abstract}

\section{Keywords}

Car Parking, Image Processing, ZigBee, GAC.

\section{INTRODUCTION}

In real time car parking, parking has been executed in many environments with various features. The smart parking system will discover the slots for parking car in parking zones. The parking zones can be developed in city areas and peculiar parking zone such as schools, universities and shopping areas [1]. The information regarding parking of cars for all types of zones will be provided by eccentric parking assistance systems [2]. There are many dimensions of problems faced in the parking place. These problems reckon on several factors.

An important factor which leads to a parking problem is human behavior. Due to time deviation in arrival rate, habitual parking lots cannot be concerned with the user. So, the users indiscriminately park the car in a parking space, and some do not have space, and they park outside of parking space. There is insentience regarding parking management in the GAC. So, we have executed a system for GAC. The image processing is used for identification purpose of the system. We identify the number plate, and we use it as the identification number of the cars.

Our consequences allocate slots to the car according to the vacancy of slots, and elite slots are allocated for VIP (Very Important Person) car, and university car, and other slots are allocated based on the request for slots and hence there is a reduction of congestion in the parking zone. Section 3 shows about ZigBee. Section 4 gives the definition for imageprocessing. Our system design is described in more in detail, and the precise rule is given in section 5 . In section 6 , performance is evaluated. A real time survey made for data collection is shown in section 7. Conclusion and Future work in section 8 .

\section{RELATED WORK}

The parking space problem has been addressed by researchers and many solutions have been produced for university campus [3]. The car parking problem is also faced in street parking in [4] and non-residential parking in city areas [5]. The parking management became as a business in many countries [6]. And thus the smart payment system is commercialized by RFID technology [7] and smart. The size and trend of parking also vary in various counties, and parking is made as a travel demand management tool. The blind spot is identified using a camera system [8].

The system implementation cost is high and tell the cars into the parking. There is an organization which has graded separating, and allocation is made as per the grade. Many of the parking systems are making commercial and in Asian cities they bring parking under government sector. In parking, there are two main approaches, one is centralized and another is a non-assisted approach. In Centralized assisted parking search (CAPS), the information processing will lay on the central processor (server). The server will collect information from a sensor in the parking spot and take decisions respectively. However, this centralized approach always has some challenges.

The Non-assisted parking search (NAPS) does not have the server, and no information will be provided to a user. The user has to wander around the parking spot for finding the vacancy of space. The slot will be allocated to the user who reaches the vacant slot first. There is a new approach called opportunistically assisted parking search (OAPS), which uses a Mobile Storage Node (MSN). The information flow is made with this MSN. This leads to increase further through its efficiency. However, the information disseminated by this not always useful. The three searching approaches are compared and the results are analyzed for finding the best. Finally, the CAPS approach shows better performance than the other two approaches in [9]. In the wireless sensor networks various types of nodes are used for identification of car [10], [11]. Sometimes searching by vacancy time is more, and hence it leads to pollution. For that purpose, genetic algorithm was programmed that increases the possibility of finding the optimum information conditions that can be translated into lower emissions of toxic greenhouse gases [12].

\section{ZIGBEE}

ZigBee is a specification for a suite of high level communication protocols [13]. The cost and power of ZigBee are a low, and it has the standardized wireless mesh network. Radio-frequency (RF) is targeted by ZigBee and the radio- 
frequency applications that require a low data rate, long battery life, and secure networking. It cost low for large-scale deployment. ZigBee technology is the standard of choice among other wireless technologies due to its efficient lowpower connectivity and ability to connect a large number of devices into a single network. The network types are star network, mesh network, cluster tree network. It has various application profiles and three different device types. The ZigBee architecture is shown in Figure 1.

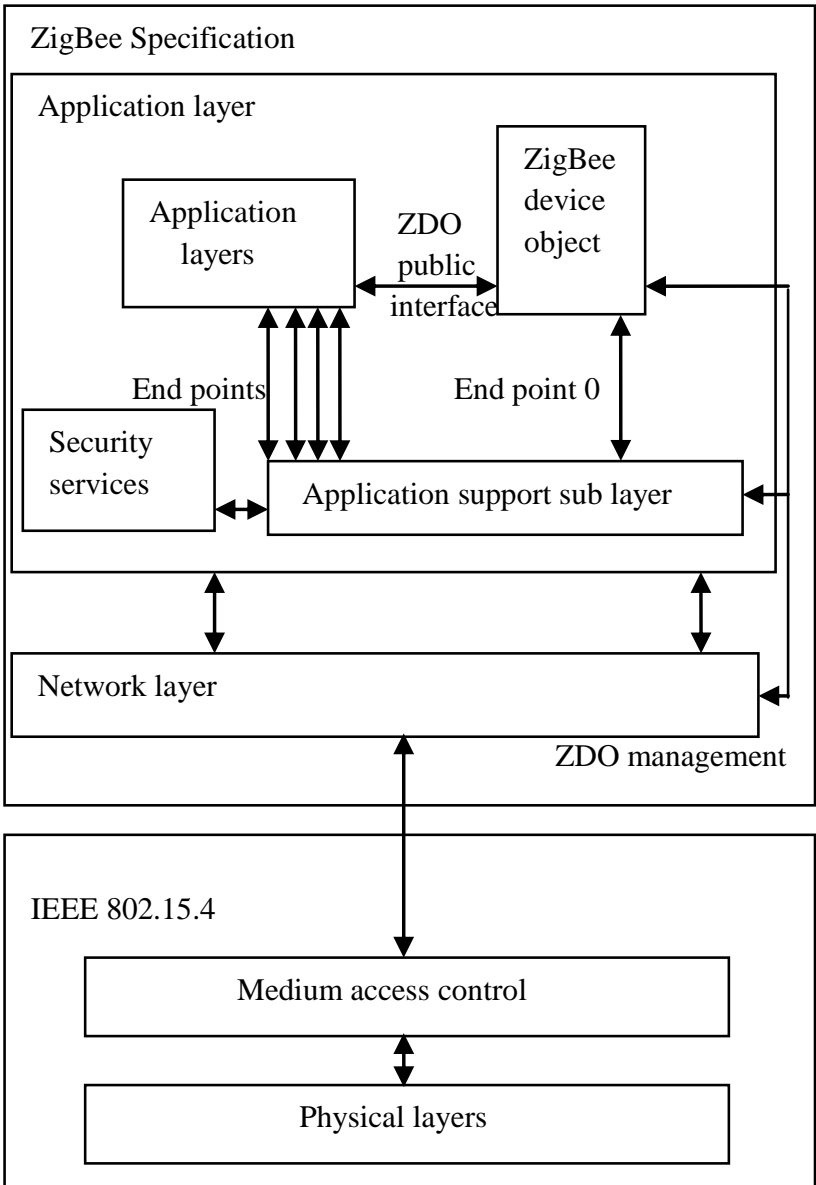

Figure 1. ZigBee Architecture

\section{IMAGE PROCESSING}

The image processing is a form of signal processing, where the input is an image or photograph, and the output can be imaged or characteristics of image or related parameters of image [14]. The image processing is used for various applications. It is usually referred as digital signal processing. The image is an array of pixel stored in a matrix format, and the pixels are initially in grayscale, and it is processed. The image processing techniques are used for identifying the car [15]. In this paper, we use this concept for finding the car number plate.

\section{PROPOSED WORK}

The Smart parking system has been contrived for GAC in an effective manner. Using ZigBee protocol and simple tools the execution is carried out for allocating the parking slots. The design of our execution is explained in the design phase, and an algorithm for allocation is given.

\subsection{Design Phase}

Our implementation is made using the ZigBee protocol and Mat lab Tool. We deploy two ZigBee protocols, one at the entrance of GAC and other at parking zone. The car number plate will be detected when a car reaches our entrance, then the detected number will be noted, and the slot will be allocated and the information will be announced through the speaker. The elite slot is determined for VIP, hence that slots will not be allocated to other cars. The information's are transmitted by ZigBee protocol.

In the parking area, the entering car will be identified by data, which is received from the protocol at the entrance. If they did not park the car in allocated slot, alert message will be provided to the user. Entry for all vehicles is made every time when enter and leave the GAC. The layout of GAC is shown below in Figure 2.

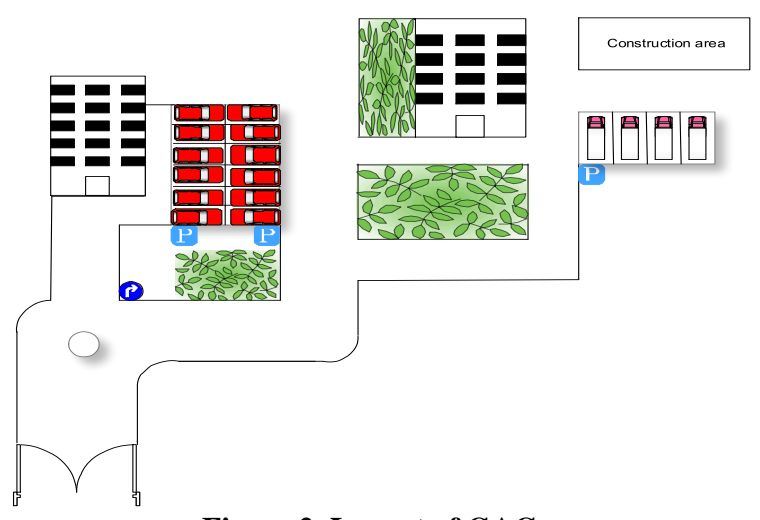

Figure 2. Layout of GAC

\subsection{Algorithm}

The Unique Identification Number (uid) is captured by the camera, and it is processed by the image processing (see in Table 1.1). Then the slot number is provided for the uid. The slot number and car number are transferred between two motes efficiently. The data is transferred before the car reaches the parking zone.

Table 1.1: Algorithm for finding uid

\section{ALGORITHM FOR FINDING UID:}

uid $\rightarrow$ unique identification number, $L \rightarrow$ Letters,

$\mathrm{N} \rightarrow$ Numbers

Let I be the set of image

Status $=\{L, N\}$

If $\mathrm{I}=1$

Get Status

Set Status to uid

The next algorithm checks, whether there is slot vacancy or not and allocates a fixed slot for VIP car numbers and remaining slots for other cars. The car number is taken as uid and it has four categories. The categories are VIP cars, 
Employee cars, Guest cars, and Construction work vehicles. For these categories, we use four slot zones. The cars other than VIP car are allocated to slots by checking their vacancy in slot zone 1 (see in Table 1.2). Then Employee cars are allocated to slot zone 2. Then Guest cars are allocated to slot zone 3. Finally, the vehicles which are used for construction works are allocated to slot zone 4. If there is no slot is left vacant, then no vacancy message is provided.

Table 1.2: Algorithm for finding vacancy

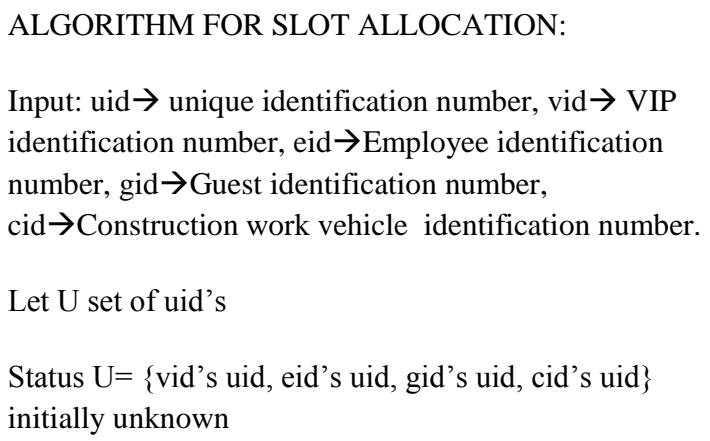

L1 allocate slot in s1

Else if status=eid 's uid go to L2

L2 allocate slot in s2

Else id status=gid's uid go to L3

L3 allocate slot in s3

Else if status=cid's uid go to L4

L4 allocate slot in s4

Set slot to uid

Else No Vacancy

End

S1 $\rightarrow$ SlotZone1; S2 $\rightarrow$ SlotZone2; S3 $\rightarrow$ SlotZone3;

$\mathrm{S} 4 \rightarrow$ SlotZone4; L1 $\rightarrow$ Label1; L2 $\rightarrow$ Label2;

L3 $\rightarrow$ Label3; L4 $\rightarrow$ Label4.

In the parking zone, the user has to park the car in the allocated slot, otherwise an alert message is produced. For producing the alert message algorithms shown in Table 1.3 is used. That algorithm has the two inputs, one is from ZigBee and other from detection of ZigBee, then the two inputs are compared, and alert message is produced.
Table 1.3: Algorithm for alert message
ALGORITHM
If $(\mathrm{rsn}==\mathrm{psn})$
Set flag $=0$
Exit
Else
Set flag $=1$
Alarm signal
Exit

Input: $r s n \rightarrow$ received slot number,

psn $\rightarrow$ parked slot number

Compare rsn and psn

\section{PERFORMANCE EVALUATION}

The Camera will take a snapshot of the number plate (shown in Figure 3), through an image processing the number plate is identified and the information is disseminated to ZigBee protocol in the parking area from ZigBee protocol in the entrance gate. The car number will be checked with the user by the alert message. Then the car reaches a parking zone, there alerts will be provided to a car if they were trying or unknowingly, parking in a slot other than what they have got allocated. The alert message will be provided to the user through the speakers. The sample number plate taken for experiment is given in following Figure 3.

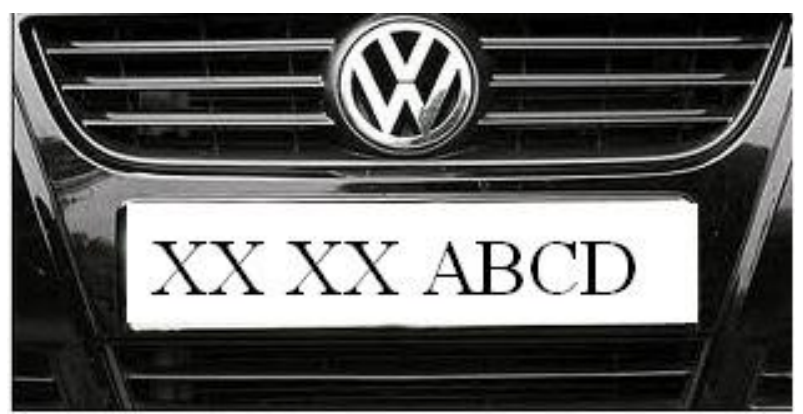

Figure 3. Sample number plate

The experimental setup is made in a laboratory with the systems, and two ZigBee protocols. As a result, the information dissemination is made, and the system in the experimental setup displays the data that was transmitted. The car number is disseminated after identification of the number plate. The experimental setup is given in Figure 4. 


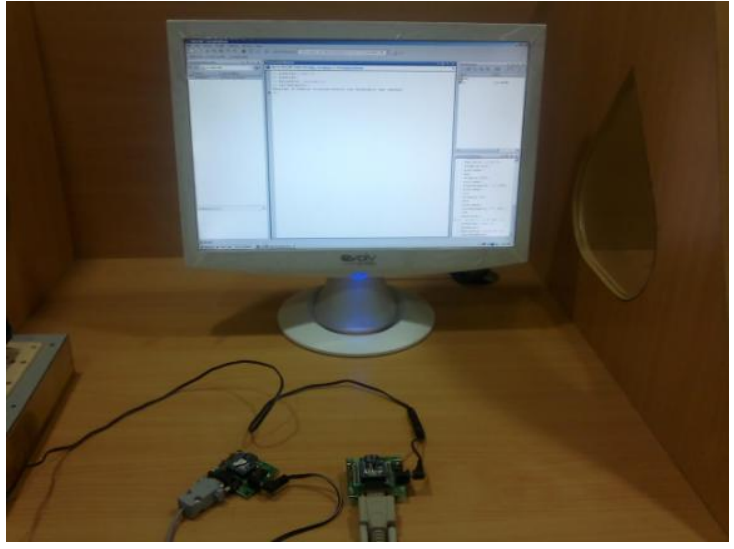

Figure 4. Experimental setup

\section{SURVEY MADE FOR COLLECTING}

\section{DATA}

The Real Time survey is done by monitoring the parking area of GAC. We assigned the slot number for the slots in the car parking area in this study. After the assignment of slots, we observe the arrival time of every car and slot number where the car has been parked, from this I encounter arrival time of some car is different and they are not homogeneous in occupying the slots. In GAC we have two areas for parking cars which are nearby and we have noticed some slots left free on one side of the parking area and congestion on the other side. To avoid this congestion we have introduced a concept of implementing a smart parking system for GAC, which was explained in detail in the previous section. Figure 5 is the graph generated based on the data collected.

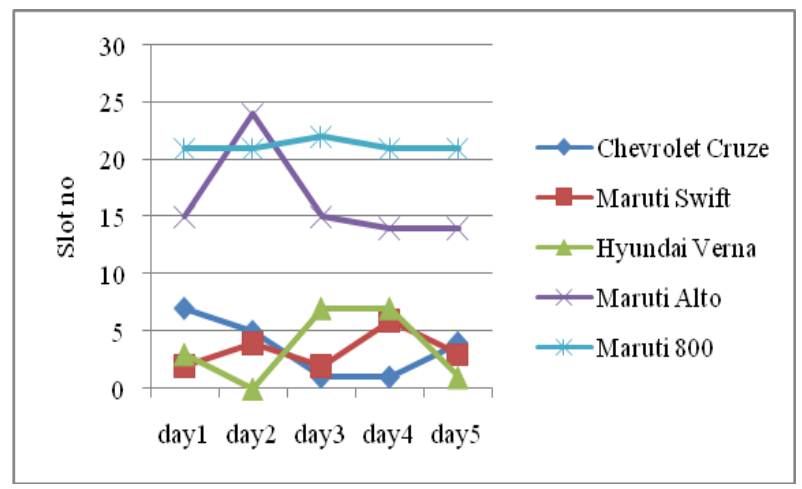

Figure 5. Parking Slot Allocation

The Table 2 is rendered into graph shown in Figure 5. The Data is collected for a particular range of period that ranges for one hour and thirty minutes. The Table 2 . contains only some of our collected data. The Table 2 . holds occupied slot number, car name and allocation percentage. The Slot numbers are fixed and it can be changed and reassigned in nature as per the requirement when there is an extension of slots. We use car name instead of giving a dummy number for graph and table. We calculate the average percentage for finding the uniformity in parking. We obtain more percentage for Maruti 800 because it tries to occupy a slot regularly. This percentage is calculated only for a limited data, where the survey contains more data. This survey is utilized for finding the possibility factor of implementation of smart car parking.
The range taken in collecting the data is one hour and thirty minutes.

Table 2: Sample data collected from parking area

\begin{tabular}{|l|l|l|}
\hline Slot No & Car & $\begin{array}{l}\text { Percentage of } \\
\text { allocation }\end{array}$ \\
\hline 1. & Chevrolet Cruze & $40 \%$ \\
\hline 2. & Maruti Swift & $40 \%$ \\
\hline 3. & Hyundai Verna & $40 \%$ \\
\hline 4. & Maruti Alto & $60 \%$ \\
\hline 5. & Maruti 800 & $80 \%$ \\
\hline
\end{tabular}

\section{CONCLUSION}

As a result, we have identified the car number plate by using image processing technique and the information is transmitted via ZigBee. This result is produced by the experimental setup made in our laboratory. And we have reduced the cost required for smart car parking when compared to previous systems what have implemented.

\section{REFERENCES}

[1] Soh Chun Khang, Teoh Jie Hong, Tan Saw Chin, Shengqiong Wang, "Wireless Mobile-Based Shopping Mall Car Parking System (WMCPS)," 2010 IEEE AsiaPacific Services Computing Conference, 2010, pp.573577.

[2] Thananchai Leephakpreeda, "Car-parking guidance with fuzzy knowledge-based decision making," Building and Environment, Volume 42, Issue 2, February 2007, pp.803-809.

[3] Eduardo Barata, Luis Cruz, João-Pedro Ferreira, "Parking at the UC campus: Problems and solutions," Cities no.28,2011,pp.406-413.

[4] Barter, P. A., "Off-street parking policy surprises in Asian cities," J. Cities., in press.

[5] Itzhak Benenson, Karel Martens, Slava Birfir, "PARKAGENT: An agent-based model of parking in the city," Computers, Environment and Urban Systems, n0.32, 2008,pp. 431-439.

[6] Chinrungrueng, J. Sunantachaikul, U. and Triamlumlerd, s., "A vehicular monitoring system with power-efficient wireless sensor networks," Proceedings of the $6^{\text {th }}$ International Conference on ITS Telecommunication, June 21-23, 2006, Chengdu, pp: 951-954.

[7] Rye, T., Ison, S.G., "Overcoming barriers to the implementation of car parking charges at UK workplaces," Transport Policy, Volume 12, Issue 1, January 2005, pp. 57-64. 
[8] Shigang Li, Ying Hai, "Easy Calibration of a Blind-SpotFree Fisheye Camera System Using a Scene of a Parking Space," Intelligent Transportation Systems, Volume 12, no. 1, march 2011, pp. 223-242.

[9] Kokolaki, E., Karaliopoulos, M., Stavrakakis, I., "Opportunistically-assisted parking service discovery: now it helps, now it does not," Elsevier Journal of Pervasive and Mobile Computing (PMC), (2011 In Press).

[10] Felix Caicedo, "Real-time parking information management to reduce search time, vehicle displacement and emissions," Transportation Research,2010,pp.228234.
[11] Benson, J.P., O’Donovan, T., O’Sullivan, P., Roedig, U., Sreenan, C., "Car-Park Management using Wireless Sensor Netwoks,” IEEE, 2006, pp. 588-595.

[12] BI Yan-Zhong, SUN Li-Min, ZHU Hong-Song, YAN Ting-Xin, LUO Zheng-Jun, "A parking management system based on wireless sensor network," ACTA AUTOMATICA SINICA 2006,Vol. 32, No. 6.

[13] ZIGBEE. http://en.wikipedia.org/wiki/ZigBee.

[14] IMAGE PROCESSING. http://en.wikipedia.org/wiki/ Image_processing.

[15] Bong, D.B.L., Ting, K.C., and Lai, K.C., "Integrated approach in the design of Car Park Occupancy Information System (COINS)," IAENG Int. J. Comput. Sci., 2008, Vol. 35, pp. 7-14. 